1 Genomic variation across a clinical Cryptococcus population linked to disease

2 outcome

4 Running title: Variants and virulence in C. neoformans

6 Poppy Sephton-Clark ${ }^{1}$, Jennifer L. Tenor ${ }^{2}$, Dena L. Toffaletti ${ }^{2}$, Nancy Meyers ${ }^{2}$, Charles

7 Giamberardino $^{2}$, Síle F. Molloy ${ }^{3,4}$, Adrienne Chan $^{5}$, Tarsizio Chikaonda ${ }^{8}$, Robert 8 Heyderman $^{6}$, Mina Hosseinipour ${ }^{7}$, Newton Kalata ${ }^{8}$, Cecilia Kanyama ${ }^{7}$, Christopher 9 Kukacha $^{8}$, Duncan Lupiya ${ }^{9}$, Henry C. Mwandumba ${ }^{8}$, Thomas Harrison ${ }^{3,4}$, Tihana 10 Bicanic $^{3,4}$, John R. Perfect ${ }^{2}$, and Christina A. Cuomo*1

$12{ }^{1}$ Infectious Disease and Microbiome Program, Broad Institute of MIT and Harvard, 13 Cambridge MA 02142 USA. 2Division of Infectious Diseases, Department of Medicine, 14 Duke University School of Medicine, Durham, NC 27710, USA. ${ }^{3}$ Centre for Global 15 Health, Institute of Infection and Immunity, St George's University of London, London, 16 United Kingdom. ${ }^{4}$ Clinical Academic Group in Infection, St George's University Hospital, 17 London, United Kingdom. ${ }^{5}$ Sunnybrook Health Sciences Centre, Toronto, Canada. $18{ }^{6}$ Division of Infection and Immunity, UCL, London, United Kingdom. ${ }^{7}$ UNC Project 19 Malawi, University of North Carolina, USA. ${ }^{8}$ Malawi-Liverpool-Wellcome Trust Clinical 20 Research Programme, Blantyre, Malawi. ${ }^{9}$ Dignitas International, Zomba, Malawi.

*Address correspondence to: Christina A. Cuomo (cuomo@broadinstitute.org)

\title{
23 Abstract
}

24 Cryptococcus neoformans is the causative agent of cryptococcosis, a disease with poor 25 patient outcomes, accounting for approximately 180,000 deaths each year. Patient 26 outcomes may be impacted by the underlying genetics of the infecting isolate, however, 27 our current understanding of how genetic diversity contributes to clinical outcomes is 28 limited. Here, we leverage clinical, in vitro growth and genomic data for $284 \mathrm{C}$. 29 neoformans isolates to identify clinically relevant pathogen variants within a population 30 of clinical isolates from patients with HIV-associated cryptococcosis in Malawi. Through 
31 a genome-wide association study (GWAS) approach, we identify variants associated 32 with fungal burden and growth rate. We also find both small and large-scale variation, 33 including aneuploidy, associated with alternate growth phenotypes, which may impact

34 the course of infection. Genes impacted by these variants are involved in transcriptional 35 regulation, signal transduction, glycolysis, sugar transport, and glycosylation. When 36 combined with clinical data, we show that growth within the CNS is reliant upon 37 glycolysis in an animal model, and likely impacts patient mortality, as CNS burden 38 modulates patient outcome. Additionally, we find genes with roles in sugar transport are 39 under selection in the majority of these clinical isolates. Further, we demonstrate that two hypothetical proteins identified by GWAS impact virulence in animal models. Our

41 approach illustrates links between genetic variation and clinically relevant phenotypes,

42 shedding light on survival mechanisms within the CNS and pathways involved in this 43 persistence.

\section{Importance}

45 Infection outcomes for cryptococcosis, most commonly caused by $C$. neoformans, are 46 influenced by host immune responses, as well as host and pathogen genetics. Infecting 47 yeast isolates are genetically diverse, however, we lack a deep understanding of how 48 this diversity impacts patient outcomes. To better understand both clinical isolate 49 diversity and how diversity contributes to infection outcome, we utilize a large collection 50 of clinical $C$. neoformans samples, isolated from patients enrolled in a clinical trial 51 across 3 hospitals in Malawi. By combining whole-genome sequence data, clinical data, 52 and in vitro growth data, we utilize genome-wide association approaches to examine the 53 genetic basis of virulence. Genes with significant associations show virulence 54 phenotypes in both murine and rabbit models, demonstrating that our approach 55 successfully identifies links between genetic variation and biologically significant 56 phenotypes.

\section{Introduction}

58 Cryptococcus neoformans is a pathogenic yeast that most commonly affects 59 immunocompromised individuals, causing an estimated 180,000 deaths annually, with 
$6075 \%$ of these occurring in sub-Saharan Africa. One of the leading causes of death in

61 adults living with HIV/AIDS, cryptococcal infections are especially problematic in low-

62 income countries where, despite a widespread roll-out of antiretroviral therapy, deaths

63 due to opportunistic infections such as cryptococcal meningitis remain high (1). The

64 infecting propagules of this pathogen generally enter human hosts via inhalation. From

65 infections within the lung, $C$. neoformans may disseminate throughout the bloodstream

66 and central nervous system of susceptible patients, causing meningitis (2). In a sample

67 of healthcare systems across low-income countries, the 1-year mortality rate for

68 individuals who develop cryptococcal meningitis is estimated to be $70 \%$ for those in

69 care (uncertainty interval 59-81\%) (1). A better understanding of $C$. neoformans strain

70 virulence and fitness within the host is necessary to improve patient outcomes and

71 develop new treatment options.

Whilst the majority of cryptococcosis cases are caused by Cryptococcus neoformans

74 var grubii (3), there are often high levels of genetic diversity within clinical populations of

75 C. neoformans (4-7). Furthermore, isolates of the same multilocus sequence type

76 (MLST) have been shown to cause infections that range in severity from mild to extreme

77 (8). To examine how genetic variation contributes to virulence phenotypes, a recent

78 study carried out logistic regression analysis with 38 clinical $C$. neoformans isolates of

79 the same sequence type to identify single nucleotide polymorphisms (SNPs) associated

80 with patient survival, clinical parameters including cytokine response, immune cell

81 counts and infection clearance, as well as in vitro data on absolute yeast growth and

82 macrophage interactions (9). This study identified 40 candidate genes based on these

83 association parameters, 6 of which (out of 17 genes tested) were important for survival

84 in a murine model of $C$. neoformans infection. In a larger cohort of 230 C. neoformans

85 samples from patients in South Africa, isolate sequence type was associated with

86 patient outcome, in vitro cerebrospinal fluid (CSF) survival, and phagocytosis response

87 (10). Full scale genome-wide association studies (GWAS) have also examined how

88 natural variation within a $C$. neoformans population differentiates clinical and

89 environmental isolates, identifying loss-of-function variants present in clinical $C$. 
neoformans (VNB) populations that impact a transcription factor important for melanization, a well-studied virulence factor (11).

Furthermore, copy number variation, such as aneuploidy, has also been frequently identified within clinical populations of $C$. neoformans. Disomy of chromosome 1 is commonly reported for isolates exposed to azoles, and the higher copy number of two key genes, the AFR1 transporter and the ERG11 drug target, confer increased resistance to antifungals such as fluconazole (12-14). Chromosome duplication as a result of in vivo passage has also been noted in clinical isolates (15-17), and the emergence of aneuploidy in this setting has been proposed as a mechanism by which both Cryptococcus and Candida species might rapidly adapt to high-stress environments $(18,19)$. In $C$. neoformans aneuploidy is often transient and passage under non-selective conditions allows for reversion to euploidy $(14,17)$. In total, aneuploidy of chromosomes $1,2,4,6,8,9,10,12,13$, and 14 have been reported in $C$. neoformans (14, 16, 17, 20-23). Despite appearing consistently throughout clinical populations, the impact of these other chromosomal aneuploidies is not yet well understood.

To better understand how genetic variation among $C$. neoformans isolates contributes to infection outcomes in patients, we carried out genome-wide association studies (GWAS) with 266 C. neoformans clinical isolates from the VNI lineage, selected to reduce the confounding effects of population structure between lineages. In addition to comparing selected clinical data, all isolates were also measured for in vitro growth under diverse conditions. Through our GWAS approach, we identify two hypothetical proteins associated with fungal burden in patients which also contribute to virulence in a murine model. Additionally, we show that growth in a rabbit model of CNS infection is dependent on glycolytic genes identified by GWAS, and corroborate findings that patient outcome is highly correlated with fungal burden in the CNS. Partial and full chromosomal duplications are commonly detected within this clinical population, yet these aneuploidies reduce $C$. neoformans fitness under in vitro growth conditions. 


\section{Materials and Methods}

\section{Sample Preparation and Sequencing}

123 Clinical cryptococcal isolates derived from patient CSF subculture were procured

124 through the Antifungal Combinations for Treatment of Cryptococcal Meningitis in Africa

125 Trial (ACTA) (24); repeat cultures and duplicates were excluded. Collected strains were

126 grown overnight in $10 \mathrm{ml}$ of $\mathrm{YPD}$ at $30^{\circ} \mathrm{C}$ and $225 \mathrm{rpm}$. Genomic DNA was then

127 extracted for sequencing with the MasterPure Yeast DNA Purification Kit, as described

128 by Desjardins et al. (11). DNA was sheared to 250bp using a Covaris LE instrument,

129 and adapted for Illumina sequencing as described by Fisher et al. (25). Libraries were

130 sequenced on a HiSeq X10, generating 150bp paired reads (minimum average

131 coverage 100x).

\section{Data Processing and Variant Calling}

134 To determine sample species, reads were first aligned to a composite pan135 Cryptococcus genome, consisting of reference genomes for Cryptococcus neoformans 136 var. grubii H99, Cryptococcus neoformans var. neoformans JEC21, and representative 137 genomes for lineages VGI, VGII, VGIIla, VGIIlb, VGIV, and VGV of Cryptococcus gattii 138 (26-29). To identify variants for $C$. neoformans species, reads were aligned to the 139 Cryptococcus neoformans var grubii H99 reference genome (GCA_000149245.3) (27) 140 with BWA-MEM version 0.7.17 (30). GATK v4 variant calling was carried out as

141 documented in our publicly available cloud-based pipeline

142 (https://github.com/broadinstitute/fungal-wdl/tree/master/gatk4). Post calling, variants 143 were filtered on the following parameters: $\mathrm{QD}<2.0$, QUAL $<30.0$, SOR $>3.0$, FS >

14460.0 (indels $>200$ ), $\mathrm{MQ}<40.0, \mathrm{GQ}<50$, alternate allele percentage $=0.8, \mathrm{DP}<10$.

145 Variants were annotated with SNPeff, version 4.3t (32).

\section{Population Genomic Analysis}

148 A maximum likelihood phylogeny was estimated using 72,258 segregating SNP sites 149 present in one or more isolates, allowing ambiguity in a maximum of $10 \%$ of samples, 150 with RAxML version 8.2.12 GTRCAT rapid bootstrapping (33), and visualized with 151 ggtree (R 3.6.0) rooted to VNII isolates. To estimate linkage disequilibrium (LD) decay, 
152 vcftools version 0.1.16 was used to calculate LD for 1000bp windows, with a minimum

153 minor allele frequency of 0.1 , and the --hap-r2 option. Region deletions and duplications

154 were identified using CNVnator v0.3 (significant instance e value < 0.01) (34). To

155 identify regions under selection, composite likelihood ratio analysis was performed with

156 PopGenome (R 3.5.0, PopGenome 2.7.5) per chromosome, by 5kb windows (35). The

157 top 5\% scoring regions (centromeric regions excluded) were tested for enrichment using

158 a hypergeometric test with FDR correction. Large duplications and aneuploidies were

159 visualized using funpipe (coverage analysis) version 0.1 .0

160 (https://github.com/broadinstitute/funpipe).

\section{Genome-Wide Association Studies}

163 Association analysis between clinical data, in vitro phenotypes, and variants was carried 164 out using PLINK v1.08p formatted files and Gemma version 0.94 .1 (36) (options: 165 centered relatedness matrix gk 1, linear mixed model), as previously described (11). 166 Variants were considered in two scenarios, one in which rare variants (present in $<5 \%$ 167 of the population) were collapsed by gene, and another in which loss-of-function 168 variants (SNPeff impact high) were considered independently. Significant variants were 169 considered to have a test score < 1.00E-6.

171 Clinical Data Analysis

172 De-identified clinical metadata detailing CSF fungal burden (CFU/ml), fungal clearance

173 (EFA), patient mortality, and Glasgow coma score were provided by investigators, with 174 these parameters determined as previously described (24). Correlation between clinical 175 parameters was determined in R 3.6.0 with Pearson correlation coefficient, Spearman's 176 rank correlation coefficient, point-biserial correlation, or Phi coefficient. Survival curves 177 were generated using Prism v9.1.0 and statistics were carried out in R 3.6.0.

179 In vitro Phenotyping

180 Strains were grown at $30^{\circ} \mathrm{C}$ for two to five days. For each strain, a single colony was 181 selected and added to 96 well microtiter plates containing $200 \mu \mathrm{L}$ of YPD broth. Each 96 182 well plate contained six control strains (H99, LAC1, MPK1, CNA1, RAS1, and HOG1) 
183 and a YPD control. The 96 well plates were incubated for one to two days at $30^{\circ} \mathrm{C}$.

184 Colonies were pin replicated into 384 well microtiter plates containing $80 \mu \mathrm{L}$ of YPD

185 broth in each well. The 384 well plates were incubated for one to two days at $30^{\circ} \mathrm{C}$.

186 They were then pinned onto one well solid agar plates in duplicate using the BM5-BC

187 Colony Processing Robot ( $\mathrm{S} \& \mathrm{P}$ Robotics) in 1536 array format. In three separate

188 biological replicates, isolates were grown at $30^{\circ} \mathrm{C}, 37^{\circ} \mathrm{C}$, and $39^{\circ} \mathrm{C}$ on YPD agar.

189 Isolates were also pinned onto YPD+10 $\mu \mathrm{g} / \mathrm{ml}$ fluconazole and YPD+64 $\mu \mathrm{g} / \mathrm{ml}$

190 fluconazole. Images were captured after approximately 24, 48, and $72 \mathrm{~h}$. Colony size at

191 48h was determined using gitter (37), and used to assess growth.

193 Gene Deletion Strains

194 Strains used for the animal studies and the primer sequences used are listed in

195 Supplemental Table 1. KN99alpha (CM026) was used as the reference wild-type strain 196 for deletions obtained from a genome-wide Cryptococcus deletion library (38). Four 197 deletion strains were generated in wild-type C. neoformans strain H99 (cnag_00544A,

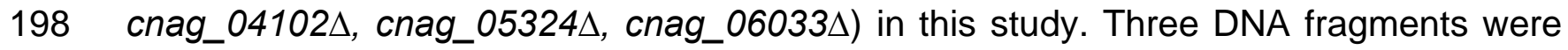
199 amplified by PCR: approximately $0.7-1 \mathrm{~kb}$ of 5 ' flank sequence, the nourseothricin 200 (NAT) drug selection cassette amplified from pAl3 (39), and 0.7-1 kb of 3' flank 201 sequence were prepared for each gene. The PCR products were gel extracted using 202 the NucleoSpin Gel and PCR Clean-up Kit (Macherey-Nagel). Next, the PCR products 203 were cloned into pUC19 using NEBuilder HiFi DNA Assembly, transformed into 204 Escherichia coli, and positive plasmids were confirmed by PCR. For biolistic 205 transformation, $2 \mu \mathrm{g}$ of plasmid was transformed into $C$. neoformans strain H99 as 206 previously described (40) with a slight modification that the cells were recovered on 207 YPD containing $0.5 \mathrm{M}$ sorbitol and $0.5 \mathrm{M}$ mannitol. The cells were allowed to recover for $2082.5 \mathrm{~h}$ before transferring to the selective medium, YPD+100 $\mu \mathrm{g} / \mathrm{ml}$ NAT. Positive 209 transformants were confirmed by PCR.

\section{Capsule production}

212 To evaluate the capsule size, capsule inducing medium (10\% Sabouraud broth in 50 $213 \mathrm{mM}$ MOPS pH 7.3) was used as previously described (41). Five milliliters of capsule 
214 inducing medium was inoculated with a single freshly streaked yeast colony and grown

215 in an incubator shaker (225 rpm) for approximately 24 and $48 \mathrm{~h}$. India ink was used as a 216 counterstain at a 1:5 ratio (ink:cell suspension). Images were captured by microscopy

217 (Zeis Axio Imager 1). Cell body and capsule size were measured in ImageJ V1.53a.

\section{Murine model of infection}

220 C. neoformans strains were grown in YPD broth at $30^{\circ} \mathrm{C}$ in a shaking incubator $(220$ $221 \mathrm{rpm}$ ) for $24 \mathrm{~h}$, centrifuged, and washed twice in phosphate-buffered saline (PBS). Cell 222 counts were determined using a T4 cell counter (Nexcelom). Five male CD-1 mice 223 (Charles River Laboratories) were infected with approximately $5 \times 10^{4}$ yeast cells per 224 mouse via oropharyngeal aspiration while under isoflurane anesthesia. Mice were 225 monitored and weighed daily. Mice with a total body weight loss of $\geq 20 \%$ or that 226 exhibited behavioral, neurological, or respiratory symptoms were sacrificed following 227 IACUC guidelines. Kaplan-Meier survival plots and analysis (the log-rank test) were 228 completed using Prism software v9.1.0.; GraphPad Software. A p-value of $\leq 0.05$ was 229 considered statistically significant.

\section{Rabbit model of infection}

232 To assess the fitness and virulence of deletion strains in rabbit CSF, 3 New Zealand 233 White male rabbits were inoculated intracisternally with $300 \mu$ of approximately $1 \times 10^{8}$ 234 cells. Animals were sedated with ketamine and xylazine for inoculation and serial CSF 235 cisternal taps. The rabbits were treated with hydrocortisone acetate $(2.5 \mathrm{mg} / \mathrm{kg}) \mathrm{by}$ 236 intramuscular injections daily starting one day prior to yeast inoculation. Cisternal taps 237 were performed on days 3, 7, and 10 followed by serial dilution of the CSF and 238 enumeration of colonies. The time series fungal burden data were then assessed by 239 using repeated measures analysis in $\mathrm{R}$ v3.6.1.

\section{$241 \quad$ Animal studies}

242 Animal experiments were performed in compliance with the Animal Welfare Act, the 243 Guide for the Care and Use of Laboratory Animals (42), and the Duke Institutional 244 Animal Care and Use Committee (IACUC). 


\section{Results}

246 The VNI lineage dominates clinical isolates and shows selection for sugar 247 transporters

248 To examine variation within clinical populations, C. neoformans samples were isolated 249 from HIV-infected patients as part of the ACTA trial (24), which evaluated the efficacy of 250 fluconazole partnered with flucytosine, compared to amphotericin B combined with 251 either fluconazole or flucytosine, as induction therapy for cryptococcal meningitis. 252 Baseline (pre-antifungal exposure) isolates were collected from three hospitals in 253 Malawi between 2013 and 2016. We performed whole-genome sequencing on 344 254 isolates and called variants against the $C$. neoformans var grubii $\mathrm{H} 99$ reference genome 255 (27). Isolates identified as Cryptococcus gattii (45), hybrid AD C. neoformans (4), diploid 256 (2), or with low coverage (9), based on evaluating read alignments to a pan257 Cryptococcus collection of reference genomes, were removed from the analysis. To 258 examine the population structure, a maximum likelihood phylogeny was built using 25972,258 segregating SNP sites (Figure 1). Isolates can be clearly identified as VNI (266), 260 VNII (9), and VNB (9) based on phylogenetic comparison with previously typed isolates 261 reported by Desjardins et al (11). VNI isolates split into VNla (217) and VNlb (49) with $262100 \%$ bootstrap support; these recently described sub-lineages show strong evidence 263 of separation (11). Of the two mating type loci found in Cryptococcus, mating type a 264 predominated among these isolates, with only one VNB isolate (ACTA3525-D) 265 possessing the alternate MATa. To assess recombination within the large VNI 266 population, we calculated linkage disequilibrium (LD) decay and found levels of decay 267 for lineage VNI (LD50 30kb) (Supplemental Figure 1) similar to those reported by 268 Desjardins et al. (LD50 for VNI, VNBI, and VNBII being < 50kb) (11). There is increased 269 decay in the VNI population as a whole when compared to individual VNla and VNIb 270 sub-groups (LD50 of 110kb for VNlb, LD50 not reached within 250kb for VNla), 271 suggesting that $\mathrm{VNla}$ and $\mathrm{VNIb}$ isolates do not recombine exclusively within their 272 groups.

274 To identify genomic regions under positive selection, we performed composite likelihood 275 ratio analysis (43). We found that regions with scores in the top $10 \%$ in more than one 
276 lineage (examining VNla, VNIb, VNII, and VNB) include subtelomeric regions, 277 centromeres, ERG11, and AFR1 (Supplemental Figure 2). To examine if genes within 278 these regions are associated with shared functions that appear under selection, we 279 performed gene ontology (GO) enrichment analysis (Hypergeometric test, FDR 280 correction) on regions with selection scores in the top 5\% (excluding centromeres). For 281 VNII isolates we found nucleotide excision repair enriched in these regions (corrected p282 value $=6.77 \mathrm{E}-3)$. Sugar transport, including inositol transport, appeared robustly 283 enriched in both VNIa and VNB lineages (corrected p-value's = 1.07E-3 and 8.60E-3), 284 supporting previous work that identified these functions as under selection 285 (Supplemental Table 2) (11). Sugar transport and utilization have been identified as key 286 to success in nutrient scarce environments such as the CNS $(23,44,45)$, during 287 interactions with amoebae, and are required for virulence and resistance to external 288 stress (46-49).

We also identified regions that were duplicated or deleted in these C. neoformans 291 isolates via copy number variation analysis (Supplemental Table 3). An $8 \mathrm{~kb}$ region was 292 found to be duplicated in $43 \mathrm{VNla}$ isolates, containing 4 genes including a sugar 293 transporter (TCDB: 2.A.1.1, glycerol transport), a predicted non-coding RNA, a fungal 294 specific transcription factor (Zn2Cys6, SIP402), and a short-chain dehydrogenase. A 295 separate $34 \mathrm{~kb}$ region was found duplicated across $48 \mathrm{VNla}$ isolates encoding 11 genes 296 including 3 dehydrogenase enzymes and 2 hydrolase enzymes. Duplicated regions 297 unique to VNlb included an un-annotated $19 \mathrm{~kb}$ region specific to 20 isolates and an $8 \mathrm{~kb}$ 298 region that encodes 2 hypothetical proteins duplicated across 21 VNIb isolates. 299 Duplications of genes involved in resistance to azoles such as ERG11 were found 300 exclusively in VNII isolates, however, this did not correlate with an enhanced ability to 301 grow in the presence of fluconazole at $64 \mathrm{ug} / \mathrm{ml}$. While these duplicated regions are not 302 directly associated with tested phenotypes, the duplication of regions containing genes 303 such as ERG11 and sugar transporters may still contribute to phenotypic variation that 304 is relevant to clinical outcomes through modulation of growth and virulence phenotypes 305 when grown in alternate conditions. 


\section{GWAS identifies multiple variants associated with fungal burden}

308 We next used clinical data associated with these isolates to investigate the relationship between clinical factors, across lineages. We confirmed previous findings that mortality correlates strongly with high baseline fungal burden (CSF CFU/ml taken at diagnosis of cryptococcal meningitis) (Figure $2 \mathrm{a}, \mathrm{p}=7.70 \mathrm{E}-7$ ) as shown in prior studies (50-52), and observed similar outcome ratios across lineages (log-rank test, $p=0.916$ ) (Figure $2 b-c$ ), suggesting no major lineage-specific differences in virulence, though low numbers of VNB and VNII infecting isolates may limit our power to detect significant differences here. Additionally, we noted similar levels of baseline fungal burden and rates of clearance between VNla and VNlb infecting isolates (Figure 2d-e). The data suggest a

317 reduction in baseline fungal burden of $1.29 \mathrm{E}+06 \mathrm{CSF} \mathrm{CFU} / \mathrm{ml}$ on average for VNII when compared to $\mathrm{VNI}$ isolates (Wilcoxon test, $\mathrm{p}=0.024$ ), however, due to the limited number of VNII isolates included, this finding should be confirmed with additional cases.

To determine whether the variation in baseline fungal burden, which appears well distributed throughout this population (Figure 1), is linked to a specific genetic component, we performed genome-wide association studies (GWAS) to identify variants associated with higher fungal load, when taken as a continuous phenotype. We selected $\mathrm{VNI}$ isolates for this analysis as they represent the major genetic group present, and to avoid confounding factors of population structure between lineages. This analysis revealed 53 variants that were significantly (GEMMA score test, $p<$ $1.00 \mathrm{E}-6)$ associated with CSF fungal burden levels (Figure 3a), 16 of which were predicted to result in a loss-of-function mutation. These variants impacted genes encoding 15 hypothetical proteins and 6 ncRNAs; an additional 4 variants fell into noncoding centromeric regions (Supplemental Table 4). Of the annotated genes impacted,

3324 have been previously identified to modulate virulence phenotypes, these include the 333 protein S-acyl transferase PFA4 (CNAG_03981), the calcineurin catalytic subunit CNA1 334 (CNAG_04796), and the mitochondrial co-chaperone MRJ1 (CNAG_00938) that are 335 required for virulence in the murine model, as well as the iron permease FTR1 336 (CNAG_06242) that is required for capsule regulation (53-56). Additionally, 2 genes 337 with variants are known to impact titan cell formation, these include the multidrug 
338 transporter CNAG_04546 and the adenylate cyclase CAC1 (CNAG_03202) $(57,58)$. A 339 high proportion (28\%) of variants with high GWAS scores (GEMMA score test, $\mathrm{p}<$ 340 1.00E-6) appeared in genes annotated as hypothetical proteins. For phenotype

341 characterization, we decided to focus on genes impacted by variants associated with 342 higher fungal burden, that were predicted to result in a loss-of-function within coding 343 regions.

\section{Two hypothetical proteins impact virulence in a murine model}

346 To determine if the genes identified through our GWAS analysis of fungal burden impact 347 virulence, we tested a total of 10 gene deletion strains across murine and rabbit models. 348 Previous work has shown that infection outcomes from human infections are well 349 recapitulated in murine models (8), while rabbit models have proven useful in evaluating 350 CNS infections, as fungal burden within the CNS can be determined through 351 longitudinal tapping of CSF (59). The most striking result from GWAS analysis was a 352 pileup of 5 variants in the hypothetical protein CNAG_04102, with the highest scoring 353 variant within this gene being the third most significant overall (GEMMA score test, $354 \mathrm{p}=1.30 \mathrm{E}-11$ ). Deletion of CNAG_04102 in a H99 background led to reduced virulence in 355 a murine model (5 male CD-1 mice), compared to a H99 isolate control (log-rank test, $356 \mathrm{p}=0.0427$ ) (Figure 3b). This gene contains a Kyakuja-Dileera-Zisupton (KDZ) 357 superfamily motif (pfam18758), which has been found within species from 358 basidiomycota, mucoromycotina, rhizophagus and allomyces (60), with CNAG_04102 359 homologs containing this motif found in Cryptococcus gattii and Cryptococcus floricola. 360 The KDZ motif is also commonly located within TET/JBP genes which are involved in 361 genomic organization and epigenetic regulation (61), suggesting a role for gene 362 expression regulation. Deletion of a second hypothetical protein, CNAG_05608, (in a 363 CMO26 KN99 background) also resulted in reduced virulence within a murine model (5 364 male CD-1 mice), with isolates lacking a functional CNAG_05608 exhibiting reduced 365 virulence, when compared to wild type (log-rank test, $p=0.0154$ ) (Figure 3c). While 366 CNAG_05608 is annotated as a hypothetical protein, this gene is predicted to contain a 367 single transmembrane domain and has homologs in Cryptococcus gattii, Cryptococcus 368 amylolentus, Kwoniella species, and Wallemia species. Furthermore, this gene is 
369 upregulated during growth in both murine and monkey lungs (62), and slightly 370 downregulated when grown in the presence of glucose (63), suggesting a role during 371 infection.

Sugar transport and metabolism impacts persistence in a rabbit CNS infection

375 When loss-of-function variants were considered independently, the most highly significant variant was a frameshift in the phosphofructokinase gene, CNAG_06033 (pfkB) (GEMMA score test, $\mathrm{p}=1.69 \mathrm{E}-09$ ). Deletion of CNAG_06033 in a H99 background resulted in significantly decreased CSF burden within the rabbit model (3 New Zealand white male rabbits) when compared to a $\mathrm{H} 99$ isolate, with CSF CFU/ml counts dependent on both the infecting strain and the number of days post-infection. The CSF loads were comparable across three rabbits for H99 and the CNAG_06033 deletion at 3 days post-infection, but decreased for the CNAG_06033 deletion at days 7 and 10, in contrast to the H99 isolate which showed increased CSF load over time

384 (repeated measures analysis, $p=0.0225$ ) (Figure 3d), highlighting the need for efficient glycolysis within the mammalian CSF (44). The overall virulence within a murine model (5 male CD-1 mice) for the CNAG_06033 deletion strain appeared similar to wild type 387 (log-rank test, $\mathrm{p}=0.1198$ ) (Figure 3e), demonstrating that defects in glycolytic metabolism do not appear to restrict persistence and dissemination but critically may be important at specific body sites. Loss-of-function variants within this gene have been shown to emerge over the course of in vivo passage in CSF during human infection and relapse (15), suggesting a role for the loss of CNAG_06033 in adaptation to the host at specific body sites. This result is consistent with prior work that tested deletions of other genes involved in glycolysis; loss of pyruvate kinase (pyk1 $\Delta$ ) resulted in decreased 394 persistence in the rabbit CSF, but unperturbed dissemination in a murine model (44).

395 Further supporting the role of glycolysis and sugar transport in the survival of $C$. neoformans in CSF, we identified significant variants in additional genes involved in

397 these pathways. A predicted xylose transporter, CNAG_05324, contained a frameshift 398 variant present in 33 isolates (GEMMA score test, $p=4.00 \mathrm{E}-07$ ). In preliminary 399 experiments deletion of CNAG_05324 in a H99 background led to an increase in CSF 
400 load in one rabbit when compared to its H99 control. However, additional experiments 401 are required to confirm these results (Supplemental Figure 3a). Given the predicted role 402 of this gene as a xylose transporter, and the presence of xylose in cryptococcal capsule, 403 we undertook preliminary phenotypic capsule screening of the CNAG_05324 deletion 404 strain. Capsule analysis of this deletion mutant via cultivation in capsule-inducing media 405 and India ink staining revealed a significant increase in capsule thickness (Welch's T406 test, $p=3.9 \mathrm{E}-14$ ) (Supplemental Figure 3b), suggesting a role for CNAG_05324 in 407 capsule size modulation. Previous work has shown that modulation of xylose transport 408 and xylosylation can drastically alter virulence, capsule size, and immune evasion (64, 409 65), highlighting this capsular mechanism as an area for further exploration.

\section{Aneuploidy is common and slows growth}

412 To determine how natural variation might affect growth and other clinically relevant 413 phenotypes, we performed in vitro phenotyping of isolates. Isolates displayed a range of 414 growth levels on rich media (YPD) at $30^{\circ} \mathrm{C}, 37^{\circ} \mathrm{C}$, and $39^{\circ} \mathrm{C}$ (Figure $4 \mathrm{a}-\mathrm{C}$ ), with colony 415 size across conditions and replicates showing strong correlation (Figure 4d,e, replicate 416 per condition $\left.\mathrm{R}^{2}>0.8\right)$. To determine whether this variation is linked to a specific 417 genetic component, we performed GWAS to identify variants associated with increased 418 and decreased colony size. Significantly associated with the rapid growth of large 419 colonies on YPD were loss-of-function variants in CNAG_06637 (UBP8 Ubiquitin420 specific protease, component of the SAGA complex), CNAG_03818 (sensory 421 transduction histidine kinase), and CNAG_10082 (tRNA Threonine) (GEMMA score 422 test, $p<1.00 \mathrm{E}-6)$. A single loss-of-function variant was found significantly associated 423 with decreased colony size, a frameshift in the dolichyldiphosphatase encoding gene, 424 CNAG_03014 (GEMMA score test, $p=9.90 E-12)$. Naturally occurring loss-of-function 425 variants such as these may play a role in the fitness variation we see between clinical 426 isolates.

428 In addition to SNP and indel mutations, we evaluated the level of chromosome copy 429 number variation across these clinical isolates. We observed both fully and partially 430 duplicated chromosomes, with the most commonly duplicated chromosomes being 12, 
4319,14 , and 1; overall, duplication of an entire chromosome occurs in $8.5 \%$ of clinical 432 isolates. Partial duplications, where at least $25 \%$ of the chromosome shows continuous 433 duplication, occur most frequently in chromosomes 2 and 6 (15 instances, Figure 5a). 434 Aneuploid isolates appear well distributed throughout this clinical dataset (Supplemental 435 Figure 4a), suggesting frequent and independent origins for these events occurring in 436 vivo.

438 To evaluate the impact of these large aneuploidies, we next compared the growth of 439 aneuploid and euploid isolates. On rich media (YPD), at $30^{\circ} \mathrm{C}, 37^{\circ} \mathrm{C}$, and $39^{\circ} \mathrm{C}$, isolates 440 harboring a fully duplicated chromosome showed significantly poorer growth than 441 euploid isolates (Wilcoxon test, $p<5.00 \mathrm{E}-07$ ), or isolates featuring only a partial 442 chromosomal duplication (Wilcoxon test, $p<0.01$ ) (Figure 5b-d). To determine whether 443 this fitness reduction occurs in both clinical and environmental populations, we carried

444 out a metanalysis of the data for these isolates, and data previously generated using the 445 same assay for a diverse set of clinical and environmental isolates (Supplemental 446 Figure 4b) (11). Isolates with aneuploidies present in both datasets displayed a 447 significant reduction in growth on YPD at $37^{\circ} \mathrm{C}(\mathrm{p}=2.00 \mathrm{E}-07)$, demonstrating that this 448 reduction in fitness holds true for both clinical and environmental isolates, across 449 lineages VNI and VNB.

Aneuploidy can be advantageous under certain stressors such as antifungal treatment, 452 however, with optimal nutrients at a range of temperatures we found that aneuploidy 453 significantly reduces fitness. Modulation of chromosome 1 ploidy has been linked to 454 apoptosis-inducing factor 1 (AIF1) (13), however, variants within AIF1 were not present 455 in this population, suggesting an alternate mechanism may be responsible for 456 modulation of chromosome copy number here.

\section{Discussion}

458 Phylogenetic analysis of 284 C. neoformans samples from patients with cryptococcosis 459 revealed a mixed population of $\mathrm{VNI}, \mathrm{VNB}$, and VNII isolates, dominated by the VNI 460 lineage. We leverage clinical metadata to identify multiple variants associated with 461 fungal burden, a factor known to impact patient mortality, in combination with additional 
462 factors such as host immune response, host genetic background, and antifungal 463 treatment. We identify both SNPs and indels significantly associated with fungal burden, 464 but not with other clinical factors such as mortality and rate of yeast clearance. We 465 utilize these association results to identify three genes involved in virulence. Of these, 466 two are annotated as hypothetical proteins and when deleted show reduced virulence in 467 a murine model, and one encodes for a phosphofructokinase that when deleted shows 468 reduced persistence in a rabbit CNS model. We employ in vitro phenotyping to identify 469 multiple variants associated with both poor and prolific growth phenotypes, and through 470 copy number analysis, we reveal multiple and varied aneuploidies within this clinical 471 population, as well as a previously reported environmental population. These 472 aneuploidies appear to reduce fitness levels in isolates from both clinical and environmental sources, across both VNI and VNB lineages.

Isolates collected from patients in Malawi as part of the ACTA study were dominated by 476 the VNI genetic group, the most commonly observed global lineage of $C$. neoformans. 477 This population consists of two of the three previously identified VNI lineages (VNIa, 478 and VNIb) $(66,67)$. VNI isolates are found around the globe and appear relatively clonal 479 when compared to the highly diverse VNB lineage, often isolated from rural niches, 480 such as mopane trees, in Africa and South America (11, 68-70).

482 Sugar transporters have previously been identified as under selection in both VNI and 483 VNB isolates from Botswana (11), and we find they also appear under selection in both 484 VNI and VNB isolates in this population from Malawi. Specifically, we find inositol, 485 xylose, glucose, lactose, and glucoside transporters under selection. The expansion of 486 inositol transporters in $C$. neoformans may offer an advantage in both woodland areas 487 and the CNS, as these environments have abundant inositol (46). Xylose transport is 488 important for $C$. neoformans capsule production, the variable xylosylation of which can 489 enable immune evasion (65). The signaling molecule and preferred carbon source 490 glucose, the precursor of glucoside, is known to regulate hexose transporters that are 491 required for virulence (47) and is a key glycolytic metabolite, a pathway required for 492 growth in the CNS (44). 
494 We identified multiple variants significantly associated with clinical and growth

495 phenotypes by taking a GWAS approach, however, clinical phenotypes such as 496 mortality and mental-status did not show a strong association with any variants 497 identified, perhaps due to the complex nature of these characteristics. Additionally, we 498 used a culture-based method to select for isolates from patients, and culture-negative 499 individuals were excluded. As a result, we were unable to detect variants associated 500 with very low levels of fungal burden in the CNS. While extensively applied to human 501 data, genome-wide association studies have also been applied to study fungal human 502 pathogens (9-11) and plant-pathogens $(71,72)$. A major challenge is adapting these 503 association approaches for the population structure of each species; for $C$. neoformans, 504 while there is recombination within the population, LD50 values for VNI, VNBI, and 505 VNBIl populations are $<50 \mathrm{~kb}$ (11). Expanding the sample size for such associations or 506 focusing narrowly on particular genetic groups can help increase the power to detect 507 variants, however, the choice of GWAS approach also needs to be optimized for the 508 population under study through consideration of population structure and size.

510 Through analysis of the clinical metadata, we found CSF fungal burden, a measure of 511 the quantity of live yeasts at the site of infection, to be the strongest factor in carrying 512 out GWAS. We found that high fungal burden within the CSF of an individual strongly 513 correlated with patient mortality, in accordance with prior work showing that high fungal 514 load is a predictor of mortality (50-52). We found that isolates lacking a functional 515 phosphofructokinase B (CNAG_06033) exhibited a reduced CSF load within a rabbit 516 model; phosphofructokinase plays a key regulatory role in the glycolytic pathway. 517 Patient isolates containing naturally occurring loss-of-function variants in CNAG_06033 518 showed no growth defects when grown on YPD at $37^{\circ} \mathrm{C}$, and our phosphofructokinase 519 mutant showed a CSF load reduction similar to that observed for the pyruvate kinase 520 (PYK1) deletion strain within a rabbit model, likely due to the similar regulatory effects of 521 both enzymes in glycolysis (44). Additionally, loss-of-function variants have previously 522 been identified in CNAG_06033 (PfkB) after in vivo human passage (15). Under stress 523 conditions, metabolically heterogeneous populations may emerge $(73,74)$, this 
524 metabolic diversity might explain the emergence of isolates less reliant upon glycolysis 525 through growth within the host. Additionally, we identified two hypothetical proteins that 526 appear to play a role in virulence within the murine model; this highlights the power of 527 our unbiased GWAS approach to systematically identify gene candidates implicated in 528 virulence, in the absence of additional functional or pathway information. Other 529 systematic studies utilizing RNA-Seq have also identified genes encoding hypothetical 530 proteins that are strong candidates for further study, due to their high expression in CSF $531(23,45)$. While the large proportion of $C$. neoformans genes annotated as hypothetical 532 proteins are more challenging to study, it is critical that we more widely characterize the 533 roles of all genes involved in the pathogenesis of $C$. neoformans.

535 We also found evidence that ploidy directly impacts the fitness of both clinical and 536 environmental C. neoformans isolates. Aneuploidy has been linked to broad-spectrum 537 stress resistance in Candida species (75), and in $C$. neoformans, disomy of 538 chromosome 1 is known to arise in isolates treated with azoles both in vitro and in vivo 539 and confers resistance to azoles such as fluconazole, through the increase in copy 540 number of $A F R 1$ and $\operatorname{ERG11}(12,14)$. Suggested mechanisms for modulation of 541 chromosome 1 ploidy include regulation via the apoptosis-inducing factor Aif1 (13), 542 however, we did not find evidence for Aif1 disruption in these isolates. Specific impacts 543 of disomy have also been noted for chromosome 13, disomy of which results in reduced 544 melanization (20). In S. cerevisiae, disomy of select chromosomes also results in 545 reduced proliferation rates (76). Whilst the reduction in fitness we observed does not 546 seem specific to any particular chromosome, the questions of how and why ploidy 547 appears subject to change in stressful conditions and whether the most frequently 548 observed aneuploidies confer a specific advantage are intriguing and will require further 549 study.

551 By combining genetic, in vitro, and clinical data, we glean insights into the impact of 552 naturally-occurring genetic variation and the implications for infection outcomes. As 553 whole genome sequencing on an ever-larger scale becomes more accessible, and 554 association techniques for microbial communities grow in sophistication, so too will our 
555 power to detect functionally relevant genetic variation across cryptococcal populations.

556 Combined with data from large pan-African clinical trials, and approaches that leverage

557 both fungal and human variant identification, we can further dissect the interaction

558 between pathogen and host genetics. Together, this will enable a better understanding

559 of how these variations impact the ability of Cryptococcus to adapt to, and thrive in, the 560 wide range of environments it finds itself within.

\section{References}

562 1. Rajasingham R, Smith RM, Park BJ, Jarvis JN, Govender NP, Chiller TM, Denning 563 DW, Loyse A, Boulware DR. 2017. Global burden of disease of HIV-associated 564 cryptococcal meningitis: an updated analysis. Lancet Infect Dis 17:873-881.

565 2. Maziarz EK, Perfect JR. 2016. Cryptococcosis. Infect Dis Clin North Am 30:179566 206.

567 3. Chayakulkeeree M, Perfect JR. 2006. Cryptococcosis. Infect Dis Clin North Am $568 \quad 20: 507-544, v-v i$.

569 4. Litvintseva AP, Thakur R, Vilgalys R, Mitchell TG. 2006. Multilocus sequence typing reveals three genetic subpopulations of Cryptococcus neoformans var. grubii (serotype A), including a unique population in Botswana. Genetics 172:2223-2238.

5. Chen Y, Litvintseva AP, Frazzitta AE, Haverkamp MR, Wang L, Fang C, Muthoga C, Mitchell TG, Perfect JR. 2015. Comparative analyses of clinical and environmental populations of Cryptococcus neoformans in Botswana. Mol Ecol

6. Nyazika TK, Hagen F, Machiridza T, Kutepa M, Masanganise F, Hendrickx M, 24:3559-3571. Robertson VJ. 2016. Cryptococcus neoformans population diversity and clinical outcomes of HIV-associated cryptococcal meningitis patients in Zimbabwe. J Med Microbiol 65:1281-1288. 
582 7. Fernandes KE, Brockway A, Haverkamp M, Cuomo CA, van Ogtrop F, Perfect JR, Carter DA. 2018. Phenotypic variability correlates with clinical outcome in Cryptococcus isolates obtained from Botswanan HIV/AIDS patients. mBio 9(5):e02016-18.

8. Mukaremera L, McDonald TR, Nielsen JN, Molenaar CJ, Akampurira A, Schutz C, Taseera K, Muzoora C, Meintjes G, Meya DB, Boulware DR, Nielsen K. 2019. The mouse inhalation model of Cryptococcus neoformans infection recapitulates strain virulence in humans and shows that closely related strains can possess differential virulence. Infection and Immunity 87(5):e00046-19.

9. Gerstein AC, Jackson KM, McDonald TR, Wang Y, Lueck BD, Bohjanen S, Smith KD, Akampurira A, Meya DB, Xue C, Boulware DR, Nielsen K. 2019. Identification of pathogen genomic differences that impact human immune response and

10. Beale MA, Sabiiti W, Robertson EJ, Fuentes-Cabrejo KM, O'Hanlon SJ, Jarvis JN, Loyse A, Meintjes G, Harrison TS, May RC, Fisher MC, Bicanic T. 2015. Genotypic diversity Is associated with clinical outcome and phenotype in Cryptococcal meningitis across southern Africa. PLOS Neglected Tropical Diseases

600 11. Desjardins CA, Giamberardino C, Sykes SM, Yu C-H, Tenor JL, Chen Y, Yang T, Jones AM, Sun S, Haverkamp MR, Heitman J, Litvintseva AP, Perfect JR, Cuomo CA. 2017. Population genomics and the evolution of virulence in the fungal pathogen Cryptococcus neoformans. Genome Research 27:1207-1219.

604 12. Sionov E, Lee H, Chang YC, Kwon-Chung KJ. 2010. Cryptococcus neoformans 605 overcomes stress of azole drugs by formation of disomy in specific multiple chromosomes. PLOS Pathogens 6(4):e1000848.

607 13. Semighini CP, Averette AF, Perfect JR, Heitman J. 2011. Deletion of Cryptococcus 608 neoformans AIF ortholog promotes chromosome aneuploidy and fluconazole- 
resistance in a metacaspase-independent manner. PLOS Pathogens 7(11):e1002364.

611 14. Stone NRH, Rhodes J, Fisher MC, Mfinanga S, Kivuyo S, Rugemalila J, Segal ES, 612 Needleman L, Molloy SF, Kwon-Chung J, Harrison TS, Hope W, Berman J, Bicanic T. 2019. Dynamic ploidy changes drive fluconazole resistance in human cryptococcal meningitis. J Clin Invest 129:999-1014.

15. Chen $\mathrm{Y}$, Farrer RA, Giamberardino C, Sakthikumar S, Jones A, Yang T, Tenor JL, Wagih O, Van Wyk M, Govender NP, Mitchell TG, Litvintseva AP, Cuomo CA, Perfect JR. 2017. Microevolution of serial clinical isolates of Cryptococcus

16. Rhodes J, Beale MA, Vanhove M, Jarvis JN, Kannambath S, Simpson JA, Ryan A, Meintjes G, Harrison TS, Fisher MC, Bicanic T. 2017. A population genomics approach to assessing the genetic basis of within-host microevolution underlying recurrent cryptococcal meningitis infection. G3: Genes, Genomes, Genetics 7:1165-1176.

624 17. Fu MS, Liporagi-Lopes LC, Dos SR, Júnior S, Tenor JL, Perfect JR, Cuomo CA, Casadevall A. 2021. Amoeba predation of Cryptococcus neoformans results in pleiotropic changes to traits associated with virulence. mBio 12(2):e00567-21.

18. Gerstein AC, Fu MS, Mukaremera L, Li Z, Ormerod KL, Fraser JA, Berman J, Nielsen K. 2015. Polyploid titan cells produce haploid and aneuploid progeny to promote stress adaptation. mBio 6(5):e01340-15.

630 19. Berman J. 2016. Ploidy plasticity: a rapid and reversible strategy for adaptation to stress. FEMS Yeast Research 16(3):fow020.

632 20. Hu G, Wang J, Choi J, Jung WH, Liu I, Litvintseva AP, Bicanic T, Aurora R, Mitchell TG, Perfect JR, Kronstad JW. 2011. Variation in chromosome copy number 634 influences the virulence of Cryptococcus neoformans and occurs in isolates from AIDS patients. BMC Genomics 12:526. 
636 21. Ormerod KL, Morrow CA, Chow EWL, Lee IR, Arras SDM, Schirra HJ, Cox GM, Fries BC, Fraser JA. 2013. Comparative genomics of serial isolates of Cryptococcus neoformans reveals gene associated with carbon utilization and virulence. G3: Genes, Genomes, Genetics 3:675-686.

22. Rhodes J, Desjardins CA, Sykes SM, Beale MA, Vanhove M, Sakthikumar S, Chen Y, Gujja S, Saif S, Chowdhary A, Lawson DJ, Ponzio V, Colombo AL, Meyer W, Engelthaler DM, Hagen F, Illnait-Zaragozi MT, Alanio A, Vreulink JM, Heitman J, Perfect JR, Litvintseva AP, Bicanic T, Harrison TS, Fisher MC, Cuomo CA. 2017. Tracing genetic exchange and biogeography of Cryptococcus neoformans var.

23. Yu C-H, Sephton-Clark P, Tenor JL, Toffaletti D, Giamberardino C, Haverkamp M, Cuomo C, Perfect J. 2021. Gene expression of diverse Cryptococcus isolates during infection of the human central nervous system. mBio 12(6):e0231321.

24. Molloy SF, Kanyama C, Heyderman RS, Loyse A, Kouanfack C, Chanda D, Mfinanga S, Temfack E, Lakhi S, Lesikari S, Chan AK, Stone N, Kalata N, Karunaharan N, Gaskell K, Peirse M, Ellis J, Chawinga C, Lontsi S, Ndong J-G, Bright P, Lupiya D, Chen T, Bradley J, Adams J, van der Horst C, van Oosterhout JJ, Sini V, Mapoure YN, Mwaba P, Bicanic T, Lalloo DG, Wang D, Hosseinipour MC, Lortholary O, Jaffar S, Harrison TS, ACTA Trial Study Team. 2018. Antifungal combinations for treatment of cryptococcal meningitis in Africa. $\mathrm{N}$ Engl $\mathrm{J}$ Med 378:1004-1017.

25. Fisher S, Barry A, Abreu J, Minie B, Nolan J, Delorey TM, Young G, Fennell TJ, Allen A, Ambrogio L, Berlin AM, Blumenstiel B, Cibulskis K, Friedrich D, Johnson R, Juhn F, Reilly B, Shammas R, Stalker J, Sykes SM, Thompson J, Walsh J, capture libraries. Genome Biology 12(1):R1. 
26. Loftus BJ, Fung E, Roncaglia P, Rowley D, Amedeo P, Bruno D, Vamathevan J, Miranda M, Anderson IJ, Fraser JA, Allen JE, Bosdet IE, Brent MR, Chiu R, Doering TL, Donlin MJ, D'Souza CA, Fox DS, Grinberg V, Fu J, Fukushima M, Haas BJ, Huang JC, Janbon G, Jones SJM, Koo HL, Krzywinski MI, Kwon-Chung JK, Lengeler KB, Maiti R, Marra MA, Marra RE, Mathewson CA, Mitchell TG, Pertea M, Riggs FR, Salzberg SL, Schein JE, Shvartsbeyn A, Shin H, Shumway M, Specht CA, Suh BB, Tenney A, Utterback TR, Wickes BL, Wortman JR, Wye NH, Kronstad JW, Lodge JK, Heitman J, Davis RW, Fraser CM, Hyman RW. 2005. The genome of the basidiomycetous yeast and human pathogen Cryptococcus neoformans. Science 307:1321-1324.

27. Janbon G, Ormerod KL, Paulet D, Byrnes EJ, Yadav V, Chatterjee G, Mullapudi N, Hon CC, Billmyre RB, Brunel F, Bahn YS, Chen W, Chen Y, Chow EWL, Coppée JY, Floyd-Averette A, Gaillardin C, Gerik KJ, Goldberg J, Gonzalez-Hilarion S, Gujja S, Hamlin JL, Hsueh YP, Ianiri G, Jones S, Kodira CD, Kozubowski L, Lam W, Marra M, Mesner LD, Mieczkowski PA, Moyrand F, Nielsen K, Proux C, Rossignol T, Schein JE, Sun S, Wollschlaeger C, Wood IA, Zeng Q, Neuvéglise C, Newlon CS, Perfect JR, Lodge JK, Idnurm A, Stajich JE, Kronstad JW, Sanyal K, Heitman J, Fraser JA, Cuomo CA, Dietrich FS. 2014. Analysis of the Genome and Transcriptome of Cryptococcus neoformans var. grubii reveals complex RNA expression and microevolution leading to virulence attenuation. PLoS Genetics 10(4):e1004261.

684 28. Farrer RA, Desjardins CA, Sakthikumar S, Gujja S, Saif S, Zeng Q, Chen Y, Voelz 685 K, Heitman J, May RC, Fisher MC, Cuomo CA. 2015. Genome evolution and innovation across the four major lineages of Cryptococcus gattii. mBio 6(5):e00868-15.

29. Farrer RA, Chang M, Davis MJ, van Dorp L, Yang DH, Shea T, Sewell TR, Meyer W, Balloux F, Edwards HM, Chanda D, Kwenda G, Vanhove M, Chang YC, Cuomo CA, Fisher MC, Kwon-Chung KJ. 2019. A new lineage of Cryptococcus gattii (VGV) discovered in the central zambezian miombo woodlands. mBio 10(6):e02306-19. 
692 30. Li H. 2013. Aligning sequence reads, clone sequences and assembly contigs with BWA-MEM. arXiv:13033997 [q-bio].

694 31. Van der Auwera GA, Carneiro MO, Hartl C, Poplin R, Del Angel G, LevyMoonshine A, Jordan T, Shakir K, Roazen D, Thibault J, Banks E, Garimella KV, Altshuler D, Gabriel S, DePristo MA. 2013. From FastQ data to high confidence variant calls: the Genome Analysis Toolkit best practices pipeline. Curr Protoc Bioinformatics 43:11.10.1-11.10.33.

32. Cingolani P, Platts A, Wang LL, Coon M, Nguyen T, Wang L, Land SJ, Lu X, Ruden DM. 2012. A program for annotating and predicting the effects of single nucleotide polymorphisms, SnpEff: SNPs in the genome of Drosophila melanogaster strain $\mathrm{w}^{1118}$; iso-2; iso-3. Fly 6:80-92.

33. Stamatakis A. 2014. RAxML version 8: a tool for phylogenetic analysis and postanalysis of large phylogenies. Bioinformatics 30:1312-1313.

34. Abyzov A, Urban AE, Snyder M, Gerstein M. 2011. CNVnator: An approach to discover, genotype, and characterize typical and atypical CNVs from family and population genome sequencing. Genome Res 21:974-984.

35. Pfeifer B, Wittelsbürger U, Ramos-Onsins SE, Lercher MJ. 2014. PopGenome: an efficient Swiss army knife for population genomic analyses in R. Mol Biol Evol 31:1929-1936.

711 36. Zhou X, Stephens M. 2012. Genome-wide efficient mixed-model analysis for association studies. 7. Nature Genetics 44:821-824.

713 37. Wagih O, Parts L. 2014. gitter: A robust and accurate method for quantification of colony sizes from plate images. G3 (Bethesda) 4:547-552.

38. Chun CD, Madhani HD. 2010. Applying genetics and molecular biology to the study of the human pathogen Cryptococcus neoformans. Methods Enzymol 470:797831. 
39. Idnurm A, Reedy JL, Nussbaum JC, Heitman J. 2004. Cryptococcus neoformans virulence gene discovery through insertional mutagenesis. Eukaryot Cell 3:420429.

40. Toffaletti DL, Rude TH, Johnston SA, Durack DT, Perfect JR. 1993. Gene transfer in Cryptococcus neoformans by use of biolistic delivery of DNA. J Bacteriol 175:1405-1411.

41. Zaragoza O, Casadevall A. 2004. Experimental modulation of capsule size in Cryptococcus neoformans. Biol Proced Online 6:10-15.

42. National Research Council (US) Committee for the Update of the Guide for the Care and Use of Laboratory Animals. 2011. Guide for the Care and Use of

43. Nielsen R, Williamson S, Kim Y, Hubisz MJ, Clark AG, Bustamante C. 2005. Genomic scans for selective sweeps using SNP data. Genome Res 15:1566-1575.

44. Price MS, Betancourt-Quiroz M, Price JL, Toffaletti DL, Vora H, Hu G, Kronstad JW, Perfect JR. 2011. Cryptococcus neoformans requires a functional glycolytic pathway for disease but not persistence in the host. mBio 2(3):e00103-e111.

45. Chen Y, Toffaletti DL, Tenor JL, Litvintseva AP, Fang C, Mitchell TG, McDonald TR, Nielsen K, Boulware DR, Bicanic T, Perfect JR. 2014. The Cryptococcus neoformans transcriptome at the site of human meningitis. mBio 5(1):e01087-13.

46. Xue C, Liu T, Chen L, Li W, Liu I, Kronstad JW, Seyfang A, Heitman J. 2010. Role of an expanded inositol transporter repertoire in Cryptococcus neoformans sexual reproduction and virulence. mBio 1(1):e00084-10.

740 47. Liu T-B, Wang Y, Baker GM, Fahmy H, Jiang L, Xue C. 2013. The glucose sensorlike protein Hxs1 Is a high-affinity glucose transporter and required for virulence in Cryptococcus neoformans. PLOS ONE 8(5):e64239. 
743 48. Li LX, Rautengarten C, Heazlewood JL, Doering TL. 2018. UDP-Glucuronic acid transport is required for virulence of Cryptococcus neoformans. mBio 9(1):e0231917.

49. Li LX, Rautengarten C, Heazlewood JL, Doering TL. 2018. Xylose donor transport is critical for fungal virulence. PLOS Pathogens 14(1):e1006765.

50. Brouwer AE, Rajanuwong A, Chierakul W, Griffin GE, Larsen RA, White NJ, Harrison TS. 2004. Combination antifungal therapies for HIV-associated cryptococcal meningitis: a randomised trial. Lancet 363:1764-1767.

51. Bicanic T, Muzoora C, Brouwer AE, Meintjes G, Longley N, Taseera K, Rebe K, Loyse A, Jarvis J, Bekker L-G, Wood R, Limmathurotsakul D, Chierakul W, Stepniewska K, White NJ, Jaffar S, Harrison TS. 2009. Independent association between rate of clearance of infection and clinical outcome of HIV-associated cryptococcal meningitis: analysis of a combined cohort of 262 patients. Clin Infect Dis 49:702-709.

52. Jarvis JN, Bicanic T, Loyse A, Namarika D, Jackson A, Nussbaum JC, Longley N, Muzoora C, Phulusa J, Taseera K, Kanyembe C, Wilson D, Hosseinipour MC, Brouwer AE, Limmathurotsakul D, White N, van der Horst C, Wood R, Meintjes G, Bradley J, Jaffar S, Harrison T. 2014. Determinants of mortality in a combined cohort of 501 patients with HIV-associated Cryptococcal meningitis: implications for improving outcomes. Clin Infect Dis 58:736-745.

53. Jung WH, Sham A, Lian T, Singh A, Kosman DJ, Kronstad JW. 2008. Iron source preference and regulation of iron uptake in Cryptococcus neoformans. PLOS Pathogens 4(2):e45. Eukaryotic Cell 14:626-635. 
769

770

771

772

773

774

775

776

777

778

779

780

781

782

783

784

785

786

787

788

789

790

791

792

793

794

795

55. Fu C, Donadio N, Cardenas ME, Heitman J. 2018. Dissecting the roles of the calcineurin pathway in unisexual reproduction, stress responses, and virulence in Cryptococcus deneoformans. Genetics 208:639-653.

56. Horianopoulos LC, Hu G, Caza M, Schmitt K, Overby P, Johnson JD, Valerius O, Braus GH, Kronstad JW. 2020. The novel J-Domain protein Mrj1 is required for mitochondrial respiration and virulence in Cryptococcus neoformans. mBio 11(3):e01127-20.

57. Okagaki LH, Wang Y, Ballou ER, O'Meara TR, Bahn Y-S, Alspaugh JA, Xue C, Nielsen K. 2011. Cryptococcal titan cell formation is regulated by G-Protein signaling in response to multiple stimuli. Eukaryot Cell 10:1306-1316.

58. Trevijano-Contador N, Oliveira HC de, García-Rodas R, Rossi SA, Llorente I, Zaballos Á, Janbon G, Ariño J, Zaragoza Ó. 2018. Cryptococcus neoformans can form titan-like cells in vitro in response to multiple signals. PLOS Pathogens 14(5):e1007007.

59. Perfect JR, Lang SD, Durack DT. 1980. Chronic cryptococcal meningitis: a new experimental model in rabbits. Am J Pathol 101:177-194.

60. Muszewska A, Steczkiewicz K, Stepniewska-Dziubinska M, Ginalski K. 2017. Cutand-paste transposons in fungi with diverse lifestyles. Genome Biology and Evolution 9:3463-3477.

61. Iyer LM, Zhang D, de Souza RF, Pukkila PJ, Rao A, Aravind L. 2014. Lineagespecific expansions of TET/JBP genes and a new class of DNA transposons shape fungal genomic and epigenetic landscapes. Proc Natl Acad Sci USA 111:16761683.

62. Li H, Li Y, Sun T, Du W, Li C, Suo C, Meng Y, Liang Q, Lan T, Zhong M, Yang S, Niu C, Li D, Ding C. 2019. Unveil the transcriptional landscape at the Cryptococcus-host axis in mice and nonhuman primates. PLOS Neglected Tropical Diseases 13(7):e0007566. 
796

797

798

799

800

801

802

803

804

805

806

807

808

809

810

811

812

813

814

815

816

817

818

819

820

821

822

63. Burke JE, Longhurst AD, Merkurjev D, Sales-Lee J, Rao B, Moresco JJ, Yates JR, Li JJ, Madhani HD. 2018. Spliceosome profiling visualizes operations of a dynamic RNP at nucleotide resolution. Cell 173:1014-1030.e17.

64. Moyrand F, Klaproth B, Himmelreich U, Dromer F, Janbon G. 2002. Isolation and characterization of capsule structure mutant strains of Cryptococcus neoformans. Mol Microbiol 45:837-849.

65. Li LX, Hole CR, Rangel-Moreno J, Khader SA, Doering TL. 2020. Cryptococcus neoformans evades pulmonary immunity by modulating xylose precursor transport. Infect Immun 88(8):e00288-20.

66. Vanhove M, Beale MA, Rhodes J, Chanda D, Lakhi S, Kwenda G, Molloy S, Karunaharan N, Stone N, Harrison TS, Bicanic T, Fisher MC. 2017. Genomic epidemiology of Cryptococcus yeasts identifies adaptation to environmental niches underpinning infection across an African HIV/AIDS cohort. Molecular Ecology 26:1991-2005.

67. Ashton PM, Thanh LT, Trieu PH, Van Anh D, Trinh NM, Beardsley J, Kibengo F, Chierakul W, Dance DAB, Rattanavong S, Davong V, Hung LQ, Chau NVV, Tung NLN, Chan AK, Thwaites GE, Lalloo DG, Anscombe C, Nhat LTH, Perfect J, Dougan G, Baker S, Harris S, Day JN. 2019. Three phylogenetic groups have driven the recent population expansion of Cryptococcus neoformans. Nature Communications 10(1):2035.

68. Litvintseva AP, Carbone I, Rossouw J, Thakur R, Govender NP, Mitchell TG. 2011. Evidence that the human pathogenic fungus Cryptococcus neoformans var. grubii may have evolved in Africa. PLoS ONE 6(5):e19688.

69. Khayhan K, Hagen F, Pan W, Simwami S, Fisher MC, Wahyuningsih R, Chakrabarti A, Chowdhary A, Ikeda R, Taj-Aldeen SJ, Khan Z, Ip M, Imran D, Sjam R, Sriburee P, Liao W, Chaicumpar K, Vuddhakul V, Meyer W, Trilles L, lersel LJJ van, Meis JF, Klaassen CHW, Boekhout T. 2013. Geographically structured 
populations of Cryptococcus neoformans variety grubii in Asia correlate with HIV status and show a clonal population structure. PLOS ONE 8:e72222.

825

826

827

828

829

830

831

832

833

834

835

836

837

838

839

840

841

842

843

844

845 75. Yang F, Teoh F, Tan ASM, Cao Y, Pavelka N, Berman J. 2019. Aneuploidy 846

70. Andrade-Silva LE, Ferreira-Paim K, Ferreira TB, Vilas-Boas A, Mora DJ, Manzato VM, Fonseca FM, Buosi K, Andrade-Silva J, Prudente B da S, Araujo NE, SalesCampos H, Silva MV da, Júnior VR, Meyer W, Silva-Vergara ML. 2018. Genotypic analysis of clinical and environmental Cryptococcus neoformans isolates from Brazil reveals the presence of VNB isolates and a correlation with biological factors. PLOS ONE 13(3):e0193237.

71. Palma-Guerrero J, Hall CR, Kowbel D, Welch J, Taylor JW, Brem RB, Glass NL. 2013. Genome wide association identifies novel loci involved in fungal communication. PLoS Genetics 9(8):e1003669.

72. Gao Y, Liu Z, Faris JD, Richards J, Brueggeman RS, Li X, Oliver RP, McDonald BA, Friesen TL. 2016. Validation of genome-wide association studies as a tool to identify virulence factors in Parastagonospora nodorum. Phytopathology 106:1177-1185.

73. Alanio A, Vernel-Pauillac F, Sturny-Leclère A, Dromer F. 2015. Cryptococcus neoformans host adaptation: toward biological evidence of dormancy. mBio 6(2):e02580-14.

74. Hommel B, Sturny-Leclère A, Volant S, Veluppillai N, Duchateau M, Yu C-H, Hourdel V, Varet H, Matondo M, Perfect JR, Casadevall A, Dromer F, Alanio A. 2019. Cryptococcus neoformans resists to drastic conditions by switching to viable but non-culturable cell phenotype. PLOS Pathogens 15(7):e1007945. enables cross-adaptation to unrelated drugs. Molecular Biology and Evolution $36: 1768-1782$. 
848 76. Torres EM, Sokolsky T, Tucker CM, Chan LY, Boselli M, Dunham MJ, Amon A. 849 2007. Effects of aneuploidy on cellular physiology and cell division in haploid yeast. Science 317:916-924.

852 Data Availability

853 Isolate sequence data can be accessed in NCBI via accession PRJNA764746.

\section{Ethics}

855 The ACTA trial from which the isolates described here were collected had ethical 856 approval from the London School of Hygiene and Tropical Medicine Research Ethics 857 Committee and by all the site national research ethics committees and regulatory 858 bodies. De-identified clinical metadata (fungal burden, fungal clearance (EFA), patient 859 outcome) was provided by investigators for analysis performed here.

\section{Acknowledgments}

861 We thank the Broad Institute Genomics Platform for generating the sequence data for 862 this study. We thank all of the patients and their families, as well as the staff at all the 863 sites not directly involved in the ACTA trial; Andrew Nunn, Halima Dawood, Andrew 864 Kitua, and William Powderly for serving on the data and safety monitoring committee; 865 Graeme Meintjes, Calice Talom, Newton Kumwenda, and Maryline Bonnet for serving 866 on the trial steering committee; and the ANRS Staff in Paris (Brigitte Bazin, Claire 867 Rekacewicz, and Paula Garcia) for constant support.

\section{Funding}

869 This project has been funded in part with Federal funds from the National Institute of 870 Allergy and Infectious Diseases, National Institutes of Health, Department of Health and 871 Human Services, under award U19Al110818 to the Broad Institute and Public Health 872 Service Grants Al73896 and Al93257 to JRP. CAC is a CIFAR fellow in the Fungal 873 Kingdom Program. RSH is a NIHR Senior Investigator. The views expressed in this 
bioRxiv preprint doi: https://doi.org/10.1101/2021.11.22.469645; this version posted November 24, 2021. The copyright holder for this preprint (which was not certified by peer review) is the author/funder, who has granted bioRxiv a license to display the preprint in perpetuity. It is made available under aCC-BY 4.0 International license.

874 publication are those of the author(s) and not necessarily those of the NIHR or the 875 Department of Health and Social Care. 


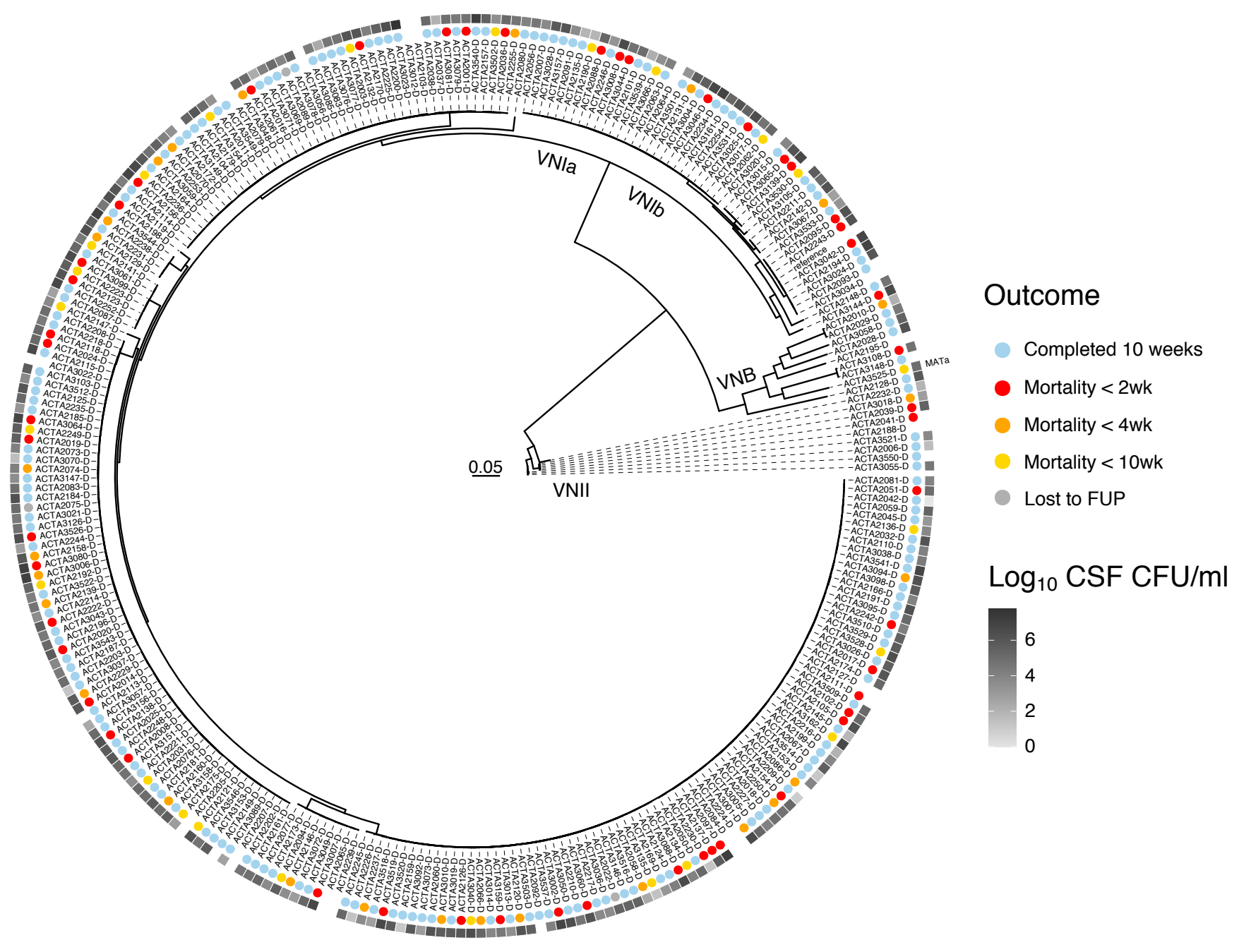

Figure 1. Maximum likelihood phylogeny of patient isolates, estimated from 72,258 segregating SNP sites, rooted to VNII. Isolates separate distinctly into VNI, VNB, and VNII, with all lineages having $100 \%$ bootstrap support. All isolates possess MAT $\alpha$ except ACTA3523 (highlighted). Colored circles correspond to patient survival. Greyscale squares indicate patient fungal burden of cerebrospinal fluid prior to treatment, $\log 10(\mathrm{CFU} / \mathrm{ml})$. 


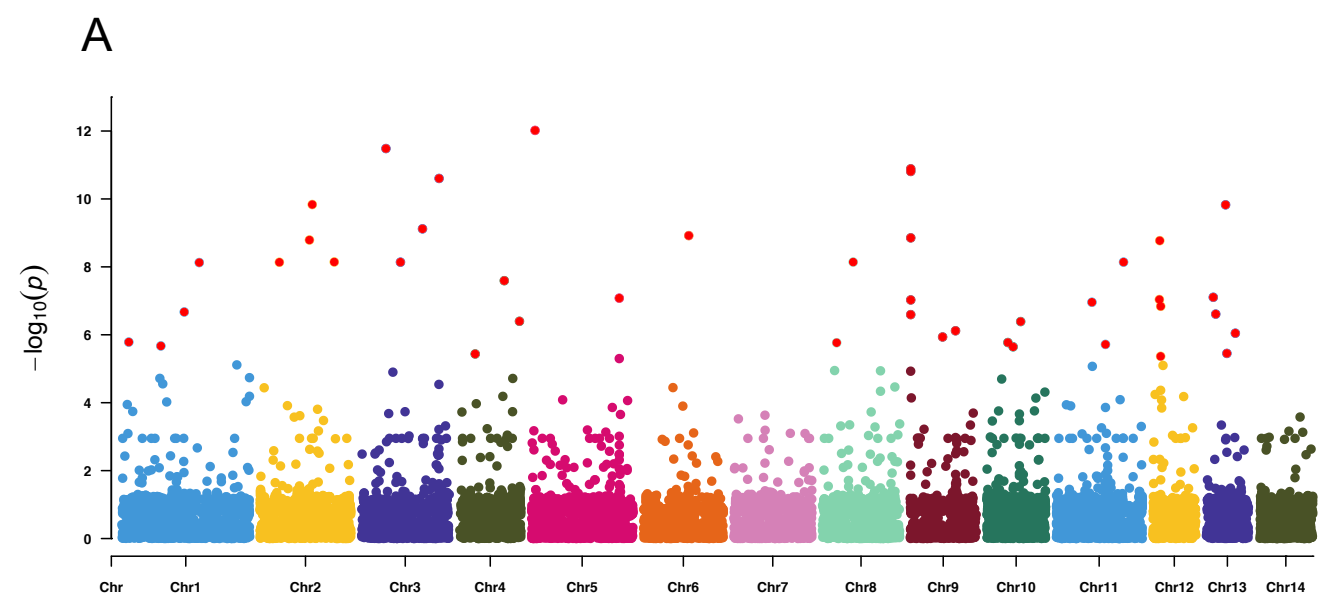

B

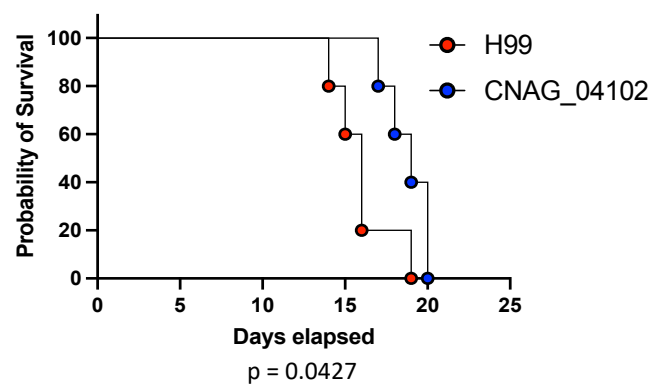

D

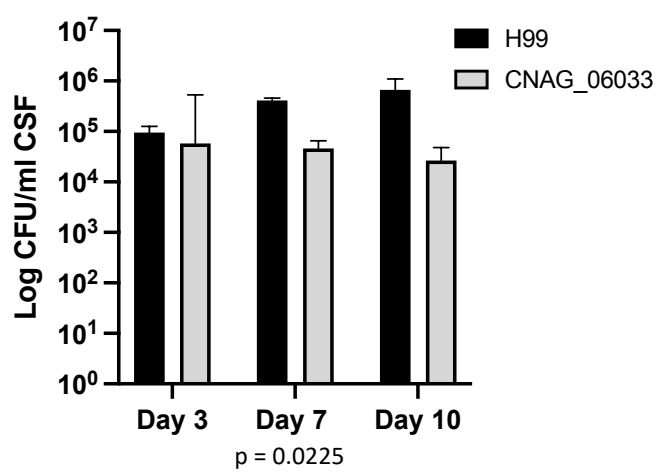

C

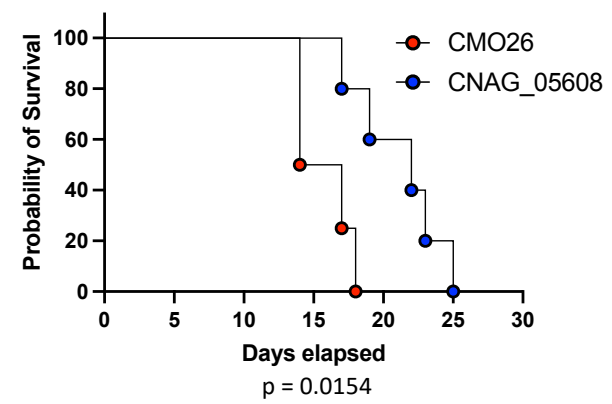

E

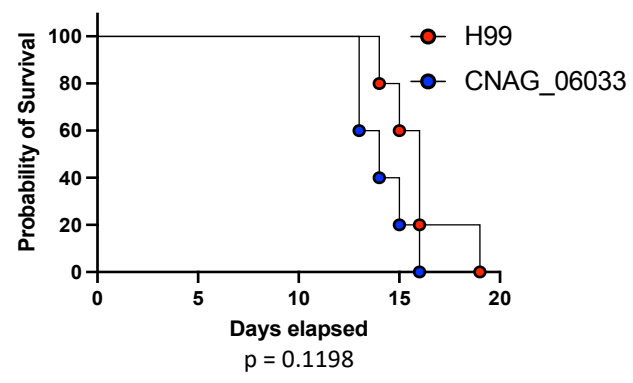

Figure 3. A) Manhattan plot displaying variants associated with high fungal burden. Variants with an association score $<0.000005$ (score test) are labeled in red. B) Survival of mice infected with parental strain (H99) and a CNAG_04102 mutant strain. Five CD-1 mice were infected with approximately 5x104 CFU by oropharyngeal aspiration. C) Survival of mice infected with parental strain (CM026) and a CNAG_05608 mutant strain. Five CD1 mice were infected with approximately $5 \times 10^{4}$ CFU by oropharyngeal aspiration. One mouse infected with CNAG_05608 was excluded from analysis as an outlier. At Day 45 of infection, this mouse was assessed for fungal burden in the lung and brain and both tissues were sterile. D) Rabbit CSF CFU's for the parental strain (H99) and a CNAG_06033 mutant strain extracted on days 3, 7, and 10 post-infection. Three rabbits were infected per strain. E) Survival of mice infected with parental strain (H99) and a CNAG_06033 mutant strain. Five CD-1 mice were infected with approximately $5 \times 10^{4}$ CFU by oropharyngeal aspiration. 

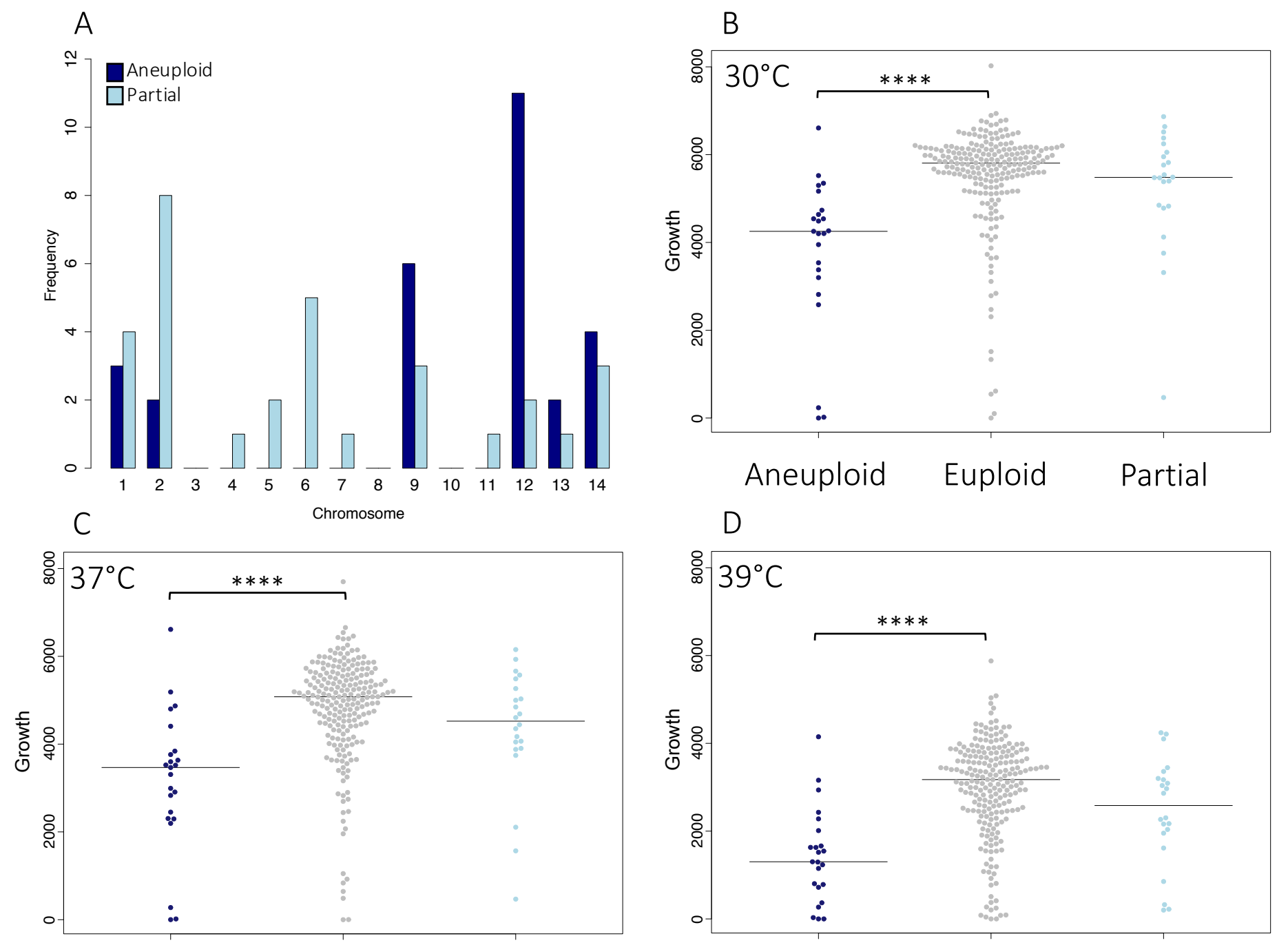

Aneuploid Euploid Partial

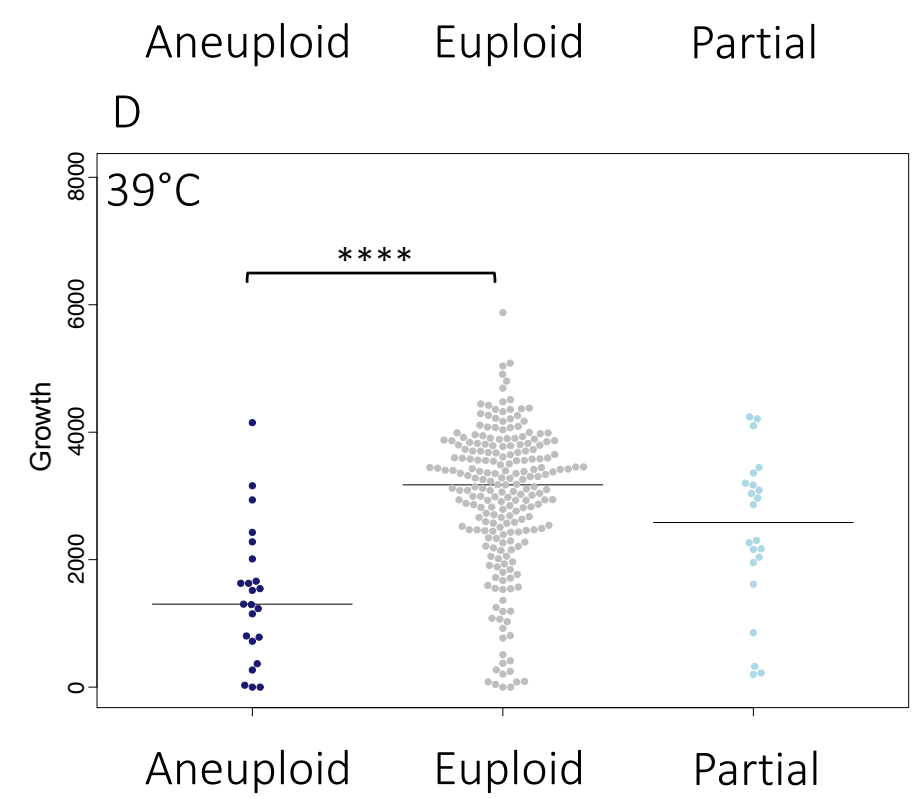

Figure 5. A) Whole (aneuploid) and partial chromosomal duplication frequency throughout the population, by chromosome. Colony size (growth) on YPD, by ploidy state at B) $\left.30^{\circ} \mathrm{C}, \mathrm{C}\right) 37^{\circ} \mathrm{C}$. D) $39^{\circ} \mathrm{C}$. 